\title{
Evolution of People Living with HIV and AIDS in Medical Consultation at 17 ESOPE Sites in Mali
}

\author{
S. Traore ${ }^{1}$, K. Kayentao ${ }^{2}$, B. Traore ${ }^{1}$, I. Coulibaly ${ }^{4}$, N. Telly ${ }^{1}$, S. Diop ${ }^{3}$, S. Doumbia ${ }^{2}$ and M. Traore ${ }^{5}$ \\ 1. Department Surveillance Epidemiology, Coordination Unit of the AIDS Control Sector Committee of the Ministry of Health and \\ Public Health (CSLS/MSHP), BPE 594, Mali
}

2. Department Parasitoly, Center for International Excellence Studies, USSTB Medical College (ICER-Mali); University Of Science,Technology of Bamako, Faculty of medicine and Odontostomalogy, Point G, Mali

3. Department of Public Health Teaching and Research, Medical and Dental College of Stomatology (DERSP), University Of Science,Technology of Bamako, Faculty of medicine and Odontostomalogy, Point G, Mali

4. Director-General of the Center for Disease Support (CNAM), Djikoroni Para, BP 251, Para, Mali

5. Director- General ofNational Institute of Public Health (INRSP), B.P. 1771, Bamako, Mali

\begin{abstract}
Despite the efforts made by the Malian government with technical and financial partners in the fight against HIV and AIDS, there are no data on the evolution of people with HIV in medical consultation in antiretroviral treatment sites. The objective of this work is to fill this gap by generating factual data describing the evolution of the number of people with HIV in medical consultation in Mali. To achieve this goal, we conducted a cross-sectional study using data from the Coordination Unit of the Sectoral Committee for AIDS Control of the Ministry of Health and Public Hygiene. Sampling was comprehensive including all people living with HIV. The data collected concerned those of the years 1999 to 2014 by the ESOPE software and analyzed with Stata version 12. A Total of 39,619 people were enrolled in the study. And 26,212 people living with HIV in the 17 ESOPE sites of Mali were female. Patients aged 25-34 and 35-44 years were strongly represented in consultation in the years 2007 and 2011, and those aged 15-24 were the least frequent. HIV1 was the most dominant with $97 \%$. The majority of patients presented for initial consultation were clinical stages 2 and 3 in all years. Cases of lost sites seen in the more common in hospitals are $62.79 \%$ at the Hospital of Mopti and 57\% at the University Hospital of Point G. The number of cases of people living with HIV in medical consultation has progressively augmented from 1999-2007. Then it dropped from 2008-2010. After this number has increased by 2011, it dropped again in 2012-2014. This study made it possible to note the evolution of the patients in medical consultation in ESOPE antiretroviral treatment sites according to the years and events (safe, subsidized by activities by the global fund). The activities of sensitization and communications, case research should be strengthened by involving the community.
\end{abstract}

Key words: Evolution, HIV, Sites of ESOPE Mali, 1999 to 2014.

\section{Introduction}

Infection with the human immunodeficiency virus (HIV) remains one of the major challenges facing global public health. To develop effective and appropriate HIV and AIDS interventions, countries need to have accurate and reliable data on HIV infection levels and trends and a better understanding of child-friendly behavioral transmission [1].

Corresponding author: Dr Sory TAORE, PharmD, MPH, research field:epidemiology. Email: sorytraore77@yahoo.fr.
In 2014, approximately 36.9 million people worldwide were infected with HIV, another 2 million were infected with HIV, and 15 million received antiretroviral therapy (ARV) [2].

In Sub-Saharan Africa, the number of people living with HIV (PLWHA) is estimated at 25.8 million, of which more than half are women. New infections have declined by 39\% from 2000 to 2014 [2].

In Mali, the free healthcare policy for all PLHIV adopted in 2004 enabled the antiretroviral treatment of 49,720 adults and 3,976 children infected by HIV. The 
number of sites supported functional (PEC) adult PHAs increased from four to 85 between 2004 and 2014 [3].

Despite the efforts made by the Malian government and the technical and financial partners in the fight against HIV and AIDS, there are no data on the evolution of the number of cases of HIV and AIDS in the various sites of antiretroviral PEC in Mali from 2004 to 2014.

The present study proposes to study the evolution of the number of cases of HIV and AIDS of the 17 sites of Evaluation and Operational Monitoring of the Esther Programs (ESOPE) of Mali from 1999 to 2014.

\section{Methodology}

We conducted a cross-sectional study that collected data retrospectively from 1999 to 2014. It was conducted at the level of the Coordination Unit of the Sector Committee for AIDS Control of the Ministry of Health and Public Hygiene (CSLS/MSHP). We conducted an exhaustive sampling including people living with HIV over the age of 15 and followed up at the 17 ESOPE sites in Mali from 1999 to 2014. The variables used were: (i) socio-demographic variables (sex, age group, and treatment sites), (ii) clinical-biological variables (HIV type, TCD4 class at initiation to antiretroviral therapy, hemoglobin rate, WHO clinical stage), (iii) variables date (year of evolution in number of people living with HIV). Data were entered into the ESOPE software from individual patient record. After they were exported in Excel format to do the cleaning and coding, the Stata software version 12 was used for statistical analysis. The Chi-two statistical test was used for the comparison of qualitative variables. We have received permission from the SHSC/SHSM coordinator to use the data anonymously to ensure confidentiality.

\section{Result}

We enrolled in the study 39,619 PLWHA. That 26,212 people living with HIV in the 17 ESOPE sites of Mali were woman (Table 1). Patients aged 35-44 and 25-34 years were highly represented in consultation in the years 2007 and 2011, and those aged 15-24 were

Table 1 Annual evolution of the number of cases of PLHIV in medical consultation in the 17 sites of ESOPE of Mali from 1999 to 2014 according to gender.

\begin{tabular}{llll}
\hline \multirow{2}{*}{ Year of consultation } & \multicolumn{2}{c}{ Sex } & Total \\
\cline { 2 - 4 } $199 *^{*}$ & Masculine & Female & 2 \\
$2000^{*}$ & 0 & 2 & 2 \\
$2001^{*}$ & 13 & 29 & 235 \\
2002 & 118 & 117 & 364 \\
2003 & 169 & 195 & 825 \\
2004 & 287 & 538 & 1,854 \\
2005 & 600 & 1,254 & 3,087 \\
2006 & 1,048 & 2,039 & 5,646 \\
2007 & 1,874 & 3,772 & 4,169 \\
2008 & 1,259 & 2,910 & 3,813 \\
2009 & 1,247 & 2,566 & 3,864 \\
2010 & 1,283 & 2,581 & 5,570 \\
2011 & 1,994 & 3,576 & 4,437 \\
2012 & 1,565 & 2,872 & 2,939 \\
2013 & 1,019 & 1,920 & 2,770 \\
2014 & 931 & 1,839 & 39,619 \\
\hline
\end{tabular}

* Represents years before the use of ESOPE in Mali but patients monitored on these dates were entered in this software. 
less frequent in the 17 sites of ESOPE of Mali (Fig. 1). HIV type 1 was the most dominant at $97 \%$ and HIV1-2 was least represented at 1\% (Fig. 2). Patients with the rate of TCD4 $\leq 200$ cells $/ \mathrm{mm}^{3}$ represented $25 \%(2,691)$ and the patients have TCD4 $\geq$ 500 cells $/ \mathrm{mm}^{3}$ represented $43 \%$ (Fig. 3). We found that patients presented themselves more in initial medical consultation at WHO clinical stages 2 and 3. The lost cases seen in the sites were more frequent in the hospitals, $62.79 \%$ at the Mopti Hospital and 57\% at the University Hospital Point G (Fig. 4).

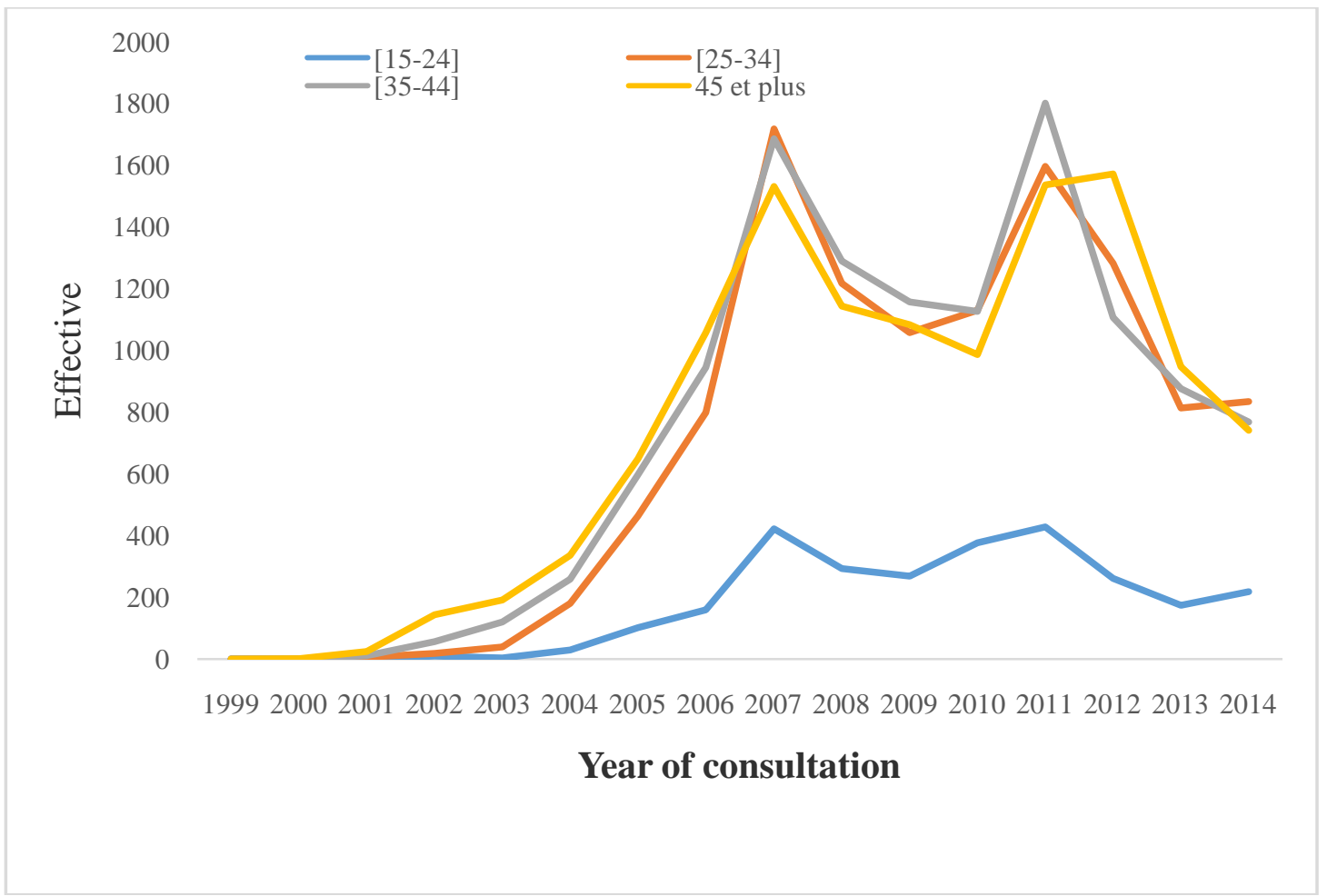

Fig. 1 Annual evolution of the number of cases of PLHIV in medical consultation in the 17 sites of ESOPE of Mali from 1999 to 2014 according to the age group.

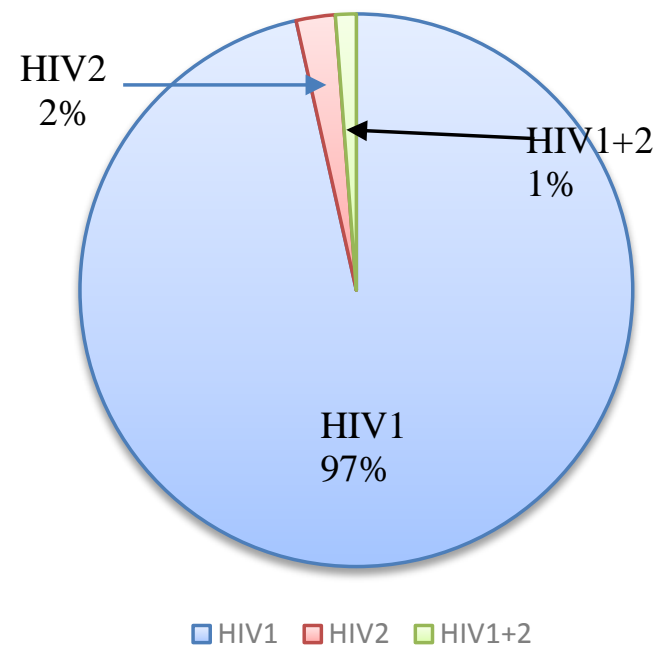

Fig. 2 Distribution of the number of cases of PLHIV and AIDS in medical consultation in the 17 sites of ESOPE of Mali from 1999 to 2014 according to the type of HIV. 


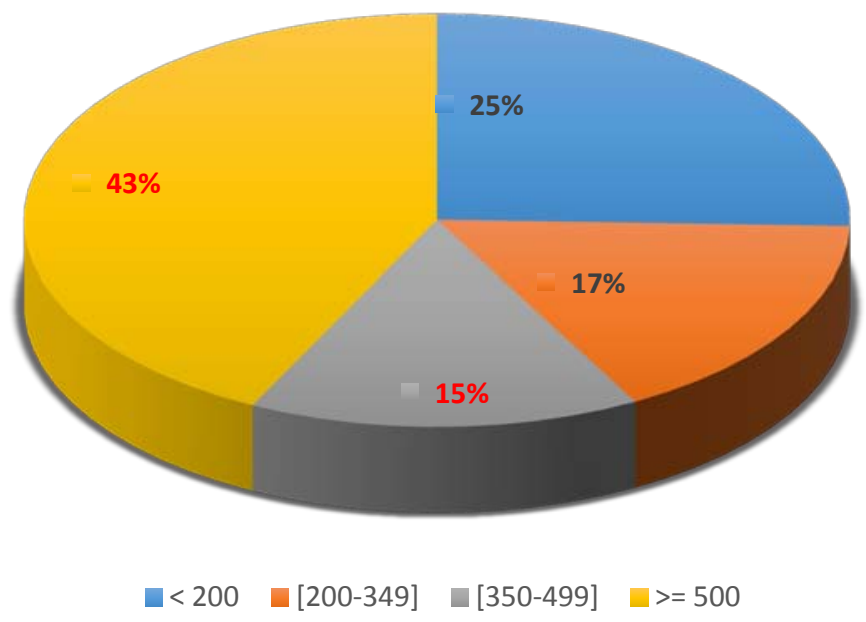

Fig. 3 Distribution of people living with HIV at 17 sites ART ESOPE of Mali, 1999 to 2014 en function of class TCD4 at inclusion ARV.

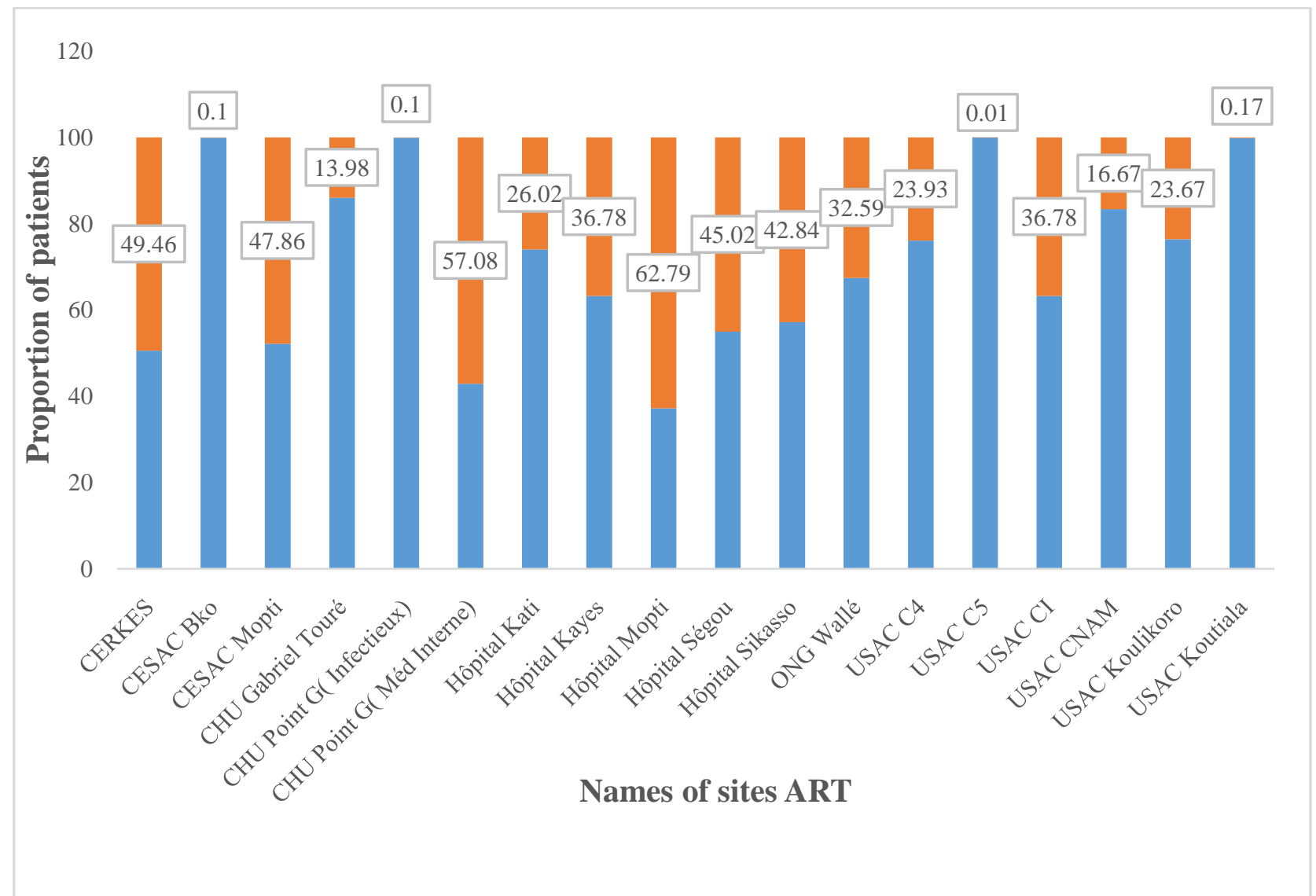

Fig. 4 Evolution of the number of cases of PLHIV and AIDS in the 17 sites of ESOPE of Mali from 1999 to 2014 according to the sites of care and by lost sight.

\section{Discussions}

Our study looked at 39,619 people living with HIV and followed up at the 17 ESOPE sites in Mali. It shows the evolution of the number of cases of people living with HIV from 1999 to 2014 according to the years in the 17 sites of retroviral management using ESOPE software. We found that $66 \%$ of the patients 
were female. This result, comparable to that obtained in Senegal by N. M. Manga, V. M. P. Ciss-Diallo, A. Lakh, M. Ndongue et al. [3], is 69\%, sex ratio female/male was 1.2, they find themselves a sex ratio of 2.3 for women. This result could be explained by the high number of females compared to males in PLWH care sites, and the high rate of female attendance.

The age group of 25-34 was the most represented in our study. In addition, another study conducted in Mali in the region of Ségou in 2008 by Ref. [4] observes the average age 34 (28-41) years. We can explain this by the fact that the most active age group is predominantly infected with HIV in the 17 sites of ESOPE in Mali, the high attendance of centers of care for PLWHA by this age group.

HIV type 1 was observed in $97 \%$ of the patients included in our study. This result is similar to that obtained in 2008 by A. Akonde, Y. Madec, A. B.Dicko, I. Haidarra, I. Katile, et al. [4] who finds $97.1 \%$. But it is lower than the result obtained in Guinea Conakry by A. Camara, B. Diaby, F. B. Sacko, A. D. Camara, H. Baldé et al. [5] observing $100 \%$ of the patients with HIV1. This difference may be due to asmallness of the sample size (429), where selection of their patients (Dermatology and Venereology Service of the National Hospital) and their study population consists only of patients with ARVs. In addition our result can be explained by the prevalence of HIV1 in the world, particularly in Mali.

About $25 \%$ of the patients included in our study had a TCD4 lymphocyte count of less than 200 cells $/ \mathrm{mm}^{3}$ at initiation of ARVs. This result is lower than that obtained in Guinea Conakry in 2008 by A. Camara et al. [5], who observed that $100 \%$ of patients had a TCD4 $<200$ cells $/ \mathrm{mm}^{3}$ when included in ARVs (Ref.[5]). This difference is explained by the small size of their sample. We can explain this result by the support of the protocol adopted CD4 count $<200$ cells $/ \mathrm{mm}^{3}$ for the eligibility of ARV in 2006, late patient resorts to care centers to benefit the dosage of TCD4 and ARV.

We observed that patients presented more in clinical stages 2 and 3 of WHO. This result is explained by the late attendance of health centers by PLHIV of the 17 ESOPE sites in Mali, the gradual and majority evolution of patients with signs of illness in the various antiretroviral treatment centers in Mali.

Our result is similar to those obtained in Congo by V. Lucaccioni et al. [6] and in Guinea by A. Camara et al. [5] who also found that the majority of patients came to clinical placement B and C.

We observed that the shadow of people living with HIV consulate in ion evolved gradually from 1999 to 2007. This can be explained by the start of the initiative to Antiretroviral Access in Mali in 2001 (IMAAR), the strong mobilization of funds by technical and financial partners especially the Global Fund, the increase in communication campaign activities for behavioral change in the face of HIV and the number of human resources trained and available to offer care quality for PLHIV, the start of free treatment of PLHIV (ARV, care, biological assessment). Moreover, this trend has decreased from 2008 to 2010. This could be due to the management problem of the global fund which has caused the scarcity and delay of the disbursement of funds for the fight against HIV. Then we observed another increase in the number of HIV cases in 2011. This result was due to the resumption of the activities of the global fund, in the fight against HIV and AIDS in the different sites of care for PLHIV by HR motivation and people tracked century sero positive to HIV. In 2014 we found a decline in the evolution of PLHIV in the 17 sites of ESOPE Mali. This decline trend could be explained by the activities of the political and security crisis, the mobilization of human resources, increasing the number of lost view of patients followed in different ART sites, reduction of activities sensitization campaigns on STIs and HIV, the abolition of certain psychological and social support activities (Income Generating Activities, culinary activities, distribution of school supplies and food at community sites), frequent break-up of ARVs and drugs against the opportunistic infections (IO), screening tests, reagents 
for biological monitoring, and other input s for the care of PLHIV.

\section{Conclusions}

From this study, it can be seen that the evolution of the number of HIV infections in ESOPE's 17 sites from 1999 to 2014 was driven by technical and financial support from Malian partners. Increase the number of human resources to take care of people living with HIV antiretroviral drugs and other drugs to opportunistic infections, free antiretroviral drugs, biological care and increase and relax propaganda activities, raise their sensitivity to sexually transmitted infections and HIV/AIDS, human resources and to stop some psychological and social support for people living with HIV, and security as well as the country's political crisis.

This result calls for new funding for HIV prevention and control activities, stabilizing the political crisis - security — and strengthening the campaign, to raise awareness of the general public and people living with HIV.

\section{References}

[1] Onu, S. "MDG 6: 15 Years, 15 Lessons of Hope from the AIDS Response.” Global Statistics 2014. UNAIDS.

[2] Unit of the Sectoral Committee for AIDS Control of the Ministry of Health and Public Hygiene of Mali. 2014. Annual Report on Antiretroviral Management of HIV and AIDS Annual Report.

[3] Manga, N. M., Ciss-Diallo, V. M. P., Lakh, A., Ndongue-Sarr, M., Batista, G., et al. "Comparative Study of PHAs under Triple Therapy with Optimal Immune Restoration and Cases ofImmuno-Virological Dissociation.” Senegal.

[4] Akonde, A., Madec, Y., Dicko, A. B., Haidarra, I.,Katile, I., Diarra, B., et al. 2008. "Decentralized Access to Triple Therapy and Viral Load Monitoring in West Africa: The Experience of the Region of Segou, Mali."

[5] Camara, A., et al. 2012. "Cauand Its Characteristics of Decius s Patients on ARVs Service Dermatology-Venereology of Donka National Hospital, Guinea.” Mali Medical 2.

[6] Lucaccioni, V., Loubaki, P., Mafoua, A., Simon, B., Mattei, J.-F., et al. 2012. "Causes of Death of HIV-Positive Patients on Antiretroviral Drugs in Developing Countries Experience of the French Red Cross Outpatient Treatment Center in Pointe-Noire, Congo.” Tropical Medicine and Health 23: 22-9. 